Louisiana State University

LSU Digital Commons

Faculty Publications

Department of Mathematics

$12-1-2007$

\title{
Moments and commutators of probability measures
}

Luigi Accardi

Università degli Studi di Roma Tor Vergata

Hui Hsiung Kuo

Louisiana State University

Aurel Stan

The Ohio State University

Follow this and additional works at: https://digitalcommons.Isu.edu/mathematics_pubs

\section{Recommended Citation}

Accardi, L., Kuo, H., \& Stan, A. (2007). Moments and commutators of probability measures. Infinite Dimensional Analysis, Quantum Probability and Related Topics, 10 (4), 591-612. https://doi.org/10.1142/ S0219025707002841

This Article is brought to you for free and open access by the Department of Mathematics at LSU Digital Commons. It has been accepted for inclusion in Faculty Publications by an authorized administrator of LSU Digital Commons. For more information, please contact ir@lsu.edu. 


\title{
MOMENTS AND COMMUTATORS OF PROBABILITY MEASURES
}

\author{
LUIGI ACCARDI \\ Centro Vito Volterra, Università di Roma "Tor Vergata", \\ 00133 Roma, Italy \\ accardi@volterra.mat.uniroma2.it \\ HUI-HSIUNG KUO \\ Department of Mathematics, Louisiana State University, \\ Baton Rouge, LA 70803, USA \\ kuo@math.lsu.edu \\ AUREL STAN \\ The Ohio State University, 1465 Mount Vernon Avenue, \\ Marion, $\mathrm{OH}$ 43302, USA \\ stan.7@osu.edu \\ Received 15 December 2005 \\ Revised 5 May 2006 \\ Communicated by O. Smolyanov
}

\begin{abstract}
Let $a^{0}, a^{-}$and $a^{+}$be the preservation, annihilation, and creation operators of a probability measure $\mu$ on $\mathbb{R}^{d}$, respectively. The operators $a^{0}$ and $\left[a^{-}, a^{+}\right]$are proven to uniquely determine the moments of $\mu$. We discuss the question: "What conditions must two families of operators satisfy, in order to ensure the existence of a probability measure, having finite moments of any order, so that, its associated preservation operators and commutators between the annihilation and creation operators are the given families of operators?" For the case $d=1$, a satisfactory answer to this question is obtained as a simple condition in terms of the Szegö-Jacobi parameters. For the multidimensional case, we give some necessary conditions for the answer to this question. We also give a table with the associated preservation and commutator between the annihilation and creation operators, for some of the classic probability measures on $\mathbb{R}$.
\end{abstract}

Keywords: Moments; creation operators; preservation operators; annihilation operators; commutators.

AMS Subject Classification: 05E35, 60H40, 81S05, 81S25

\section{Introduction}

In the paper ${ }^{2}$ we have characterized the polynomially symmetric and polynomially factorizable probability measures on $\mathbb{R}^{d}$ in terms of their associated operators: 
(1) the preservation operators $\left\{a^{0}(i) ; 1 \leq i \leq d\right\}$,

(2) the annihilation operators $\left\{a^{-}(i) ; 1 \leq i \leq d\right\}$, and

(3) the creation operators $\left\{a^{+}(i) ; 1 \leq i \leq d\right\}$.

In Ref. 2, the operator $a^{0}$ was called the number operator. Since in the centered Gaussian case $a^{0}=0$ and there exists a well-known nonzero number operator $N$, we will call $a^{0}$, the preservation (neutral) operator, from now on, in order to avoid any confusion.

In Theorem 3.1 of Ref. 2 , it is shown that a probability measure $\mu$ is polynomially symmetric if and only if the associated preservation operators $a^{0}(i), 1 \leq i \leq d$, are zero. On the other hand, it follows from Theorem 4.5 of Ref. 2 that a probability measure $\mu$ is polynomially factorizable if and only if for all $i \neq j$, any operator from the set $\left\{a^{0}(i), a^{+}(i), a^{-}(i)\right\}$ commutes with any operator from the set $\left\{a^{0}(j), a^{+}(j), a^{-}(j)\right\}$. This condition is equivalent to the fact that for all $i \neq j$, the operator $a^{0}(i)$ commutes with the operator $X_{j}$ of multiplication by the coordinate variable $x_{j}$.

In this paper we will focus on two types of operators, namely, the preservation operators $\left\{a^{0}(i)\right\}_{1 \leq i \leq d}$ and the commutators between the annihilation and creation operators $\left\{\left[a^{-}(j), a^{+}(k)\right]\right\}_{1 \leq j, k \leq d}$. We will show that the information about the moments of a probability measure on $\mathbb{R}^{d}$ is completely determined by these two types of operators. Moreover, we will consider the following question:

"Given two families of operators, does there exist a probability measure $\mu$ such that the preservation operators and the commutators between the creation and annihilation operators of $\mu$ are the given families of operators?"

We will derive some necessary conditions, in order for the answer of this question to be "Yes". Finally, we will give some tables to provide information on these two types of operators for some well-known probability measures on $\mathbb{R}$.

\section{Fundamental Identities}

In this section we will briefly review some known facts about the preservation, annihilation, and creation operators. These operators will be referred to as the PAC operators.

Let $\mu$ be a probability measure on the Borel subsets of $\mathbb{R}^{d}$ having finite moments of any order, i.e. for all $1 \leq p<\infty$ and $1 \leq j \leq d$,

$$
\int_{\mathbb{R}^{d}}\left|x_{j}\right|^{p} \mu(d x)<\infty
$$

where $x=\left(x_{1}, x_{2}, \ldots, x_{d}\right) \in \mathbb{R}^{d}$.

Let $\mathcal{P}_{0}=\mathbb{C}$. For $n \in \mathbb{N}$, let $\mathcal{P}_{n}$ be the complex vector space of all polynomial functions of $x_{1}, x_{2}, \ldots, x_{d}$, of degree less than or equal to $n$. Obviously, we have the inclusions:

$$
\mathbb{C}=\mathcal{P}_{0} \subset \mathcal{P}_{1} \subset \mathcal{P}_{2} \subset \cdots \subset \mathcal{P}_{n} \subset \cdots \subset L^{2}\left(\mathbb{R}^{d}, \mu\right) .
$$


To be completely rigorous, for the last inclusion from (2.1) to make sense, if $\mu$ has finite support, then for all $n \geq 0, \mathcal{P}_{n}$ denotes the space $V_{n}$ of polynomial functions of degree at most $n$, modulo the $\mu$-almost sure equality (which is an equivalence relation). That means, if $\mu$ is a finite convex combination of Dirac delta measures, then two polynomials that are $\mu$-almost surely equal, are considered to be the same.

Let $G_{0}=\mathbb{C}$ and for $n \in \mathbb{N}$, let $G_{n}=\mathcal{P}_{n} \ominus \mathcal{P}_{n-1}$, i.e. the orthogonal complement of $\mathcal{P}_{n-1}$ in $\mathcal{P}_{n}$. We denote by $\mathcal{H}$ the orthogonal direct sum of $G_{n}, n \geq 0$ :

$$
\mathcal{H}=\bigoplus_{n \geq 0} G_{n} \quad \text { Hilbert space sense }
$$

For each $1 \leq j \leq d$, let $X_{j}$ denote the multiplication operator by $x_{j}$. This operator is densely defined on $\mathcal{H}$ and its domain contains $\mathcal{P}_{n}$ for all $n \geq 0$. Moreover, $X_{j}$ maps $\mathcal{P}_{n}$ into $\mathcal{P}_{n+1}$, for any $n \geq 0$.

Let $P_{n}$ denote the orthogonal projection of $\mathcal{H}$ onto $G_{n}$. The following theorems are due to Accardi and Nahni, ${ }^{4}$ see also Ref. 2.

Theorem 2.1. For any $j \in\{1,2, \ldots, d\}$ and $n \geq 0$, we have

$$
X_{j} G_{n} \perp G_{k}, \quad \forall k \neq n-1, n, n+1 .
$$

Theorem 2.2. (Recursion relations) For any $j \in\{1,2, \ldots, d\}$ and $n \geq 0$, the following equality holds:

$$
X_{j} P_{n}=P_{n+1} X_{j} P_{n}+P_{n} X_{j} P_{n}+P_{n-1} X_{j} P_{n},
$$

where $P_{-1}=0$ by convention.

For each $j \in\{1,2, \ldots, d\}$ and $n \geq 0$, we define the operators:

$$
\begin{aligned}
& D_{n}^{+}(j)=P_{n+1} X_{j} P_{n}: G_{n} \rightarrow G_{n+1}, \\
& D_{n}^{0}(j)=P_{n} X_{j} P_{n}: G_{n} \rightarrow G_{n}, \\
& D_{n}^{-}(j)=P_{n-1} X_{j} P_{n}: G_{n} \rightarrow G_{n-1},
\end{aligned}
$$

where $G_{-1}=\{0\}$ by convention.

Theorem 2.3. For any $i, j \in\{1,2, \ldots, d\}$ and $n \geq 0$, the following identities hold:

$$
\begin{gathered}
\text { (a) } D_{n+1}^{+}(i) D_{n}^{+}(j)=D_{n+1}^{+}(j) D_{n}^{+}(i) \\
\text { (b) } D_{n+1}^{0}(i) D_{n}^{+}(j)+D_{n}^{+}(i) D_{n}^{0}(j) \\
\quad=D_{n+1}^{0}(j) D_{n}^{+}(i)+D_{n}^{+}(j) D_{n}^{0}(i) \\
\text { (c) } D_{n+1}^{-}(i) D_{n}^{+}(j)+D_{n}^{0}(i) D_{n}^{0}(j)+D_{n-1}^{+}(i) D_{n}^{-}(j) \\
\quad=D_{n+1}^{-}(j) D_{n}^{+}(i)+D_{n}^{0}(j) D_{n}^{0}(i)+D_{n-1}^{+}(j) D_{n}^{-}(i) .
\end{gathered}
$$


Theorem 2.4. For any $j \in\{1,2, \ldots, d\}$ and $n \geq 0$, the PAC operators $D_{n}^{0}(j)$, $D_{n}^{-}(j), D_{n}^{+}(j)$ satisfy the identities:

$$
\begin{aligned}
& \left(D_{n}^{0}(j)\right)^{*}=D_{n}^{0}(j), \\
& \left(D_{n}^{+}(j)\right)^{*}=D_{n+1}^{-}(j) .
\end{aligned}
$$

For each $j \in\{1,2, \ldots, d\}$, we consider the densely defined linear operators $a^{+}(j)$, $a^{0}(j)$ and $a^{-}(j)$ on $\mathcal{H}$, so that, for any $n \geq 0$,

$$
\left.a^{+}(j)\right|_{G_{n}}=D_{n}^{+}(j),\left.\quad a^{0}(j)\right|_{G_{n}}=D_{n}^{0}(j),\left.\quad a^{-}(j)\right|_{G_{n}}=D_{n}^{-}(j) .
$$

By Eq. (2.3), the multiplication operator $X_{j}$ can be rewritten as

$$
X_{j}=a^{+}(j)+a^{0}(j)+a^{-}(j), \quad 1 \leq j \leq d .
$$

Thus, associated with a probability measure $\mu$, having finite moments of any order, we have an interacting Fock space

$$
\left\{\mathcal{H}, 1, a^{+}(j), a^{0}(j), a^{-}(j) ; 1 \leq j \leq d\right\} .
$$

Here the constant function 1 is called the vacuum vector. Notice that $a^{-}(j) 1=0$ for any $j \in\{1,2, \ldots, d\}$.

\section{Moment Equal Probability Measures}

In this section we will see that a probability measure on $\mathbb{R}^{d}$ is completely characterized, in the sense of moments, by the operators $\left\{a^{0}(i)\right\}_{1 \leq i \leq d}$ and commutators $\left\{\left[a^{-}(j), a^{+}(k)\right]\right\}_{1 \leq j, k \leq d}$.

Definition 3.1. Two probability measures $\mu$ and $\nu$ on $\mathbb{R}^{d}$, having finite moments of any order, are said to be moment equal if they have the same moments. That means, for any monomial $m(x)=x_{1}^{i_{1}} \cdots x_{d}^{i_{d}}$, we have:

$$
\int_{\mathbb{R}^{d}} m(x) \mu(d x)=\int_{\mathbb{R}^{d}} m(x) \nu(d x) .
$$

If $\mu$ and $\nu$ are two probability measures on $\mathbb{R}^{d}$, having finite moments of any order, then we say that $\mu \stackrel{m}{=} \nu$ if $\mu$ and $\nu$ are moment equal. It is clear that $\stackrel{m}{=}$ is an equivalence relation on the set, $\mathcal{M}\left(\mathbb{R}^{d}\right)$, of all probability measures on $\mathbb{R}^{d}$, having finite moments of any order.

For any probability measure $\mu$ on $\mathbb{R}^{d}$, having finite moments of any order, we introduce two matrices $A_{\mu}^{0}$ and $A_{\mu}^{-,+}$, whose entries are operators defined on the space $\mathcal{P}$, which is the space $V$, of all polynomial functions of $d$ variables, factorized by the $\mu$-almost sure equality. Namely, $A_{\mu}^{0}$ is the column matrix, whose $i$-entry is $a^{0}(i)$, for $1 \leq i \leq d$, and $A_{\mu}^{-,+}$is the $d \times d$ matrix, whose $(j, k)$-entry is $\left[a^{-}(j), a^{+}(k)\right]$, where $\left[a^{-}(j), a^{+}(k)\right]:=a^{-}(j) a^{+}(k)-a^{+}(k) a^{-}(j)$ is the commutator of the operators $a^{-}(j)$ and $a^{+}(k)$, for $1 \leq j, k \leq d$. 
Theorem 3.2. (Fundamental Theorem) Two probability measures $\mu$ and $\nu$ on $\mathbb{R}^{d}$, having finite moments of any order, are moment equal if and only if $A_{\mu}^{0}=A_{\nu}^{0}$ and $A_{\mu}^{-,+}=A_{\nu}^{-,+}$.

Proof. $(\Rightarrow)$ Let us assume that $\mu$ and $\nu$ are moment equal. Applying the GramSchmidt orthogonalization procedure to the set of all monomials, listed in a nondecreasing degree order and arranging the monomials of the same degree in a lexicographic order, we obtain the same complete orthonormal set for the space $\mathcal{P}$, with respect to $\mu$ and $\nu$. This is true, because the coefficients in the GramSchmidt orthogonalization procedure are moments, which are the same for $\mu$ and $\nu$. Due to this fact, the spaces $G_{n}, n \geq 0$, and their bases obtained by this procedure are the same for $\mu$ and $\nu$. Thus the projections $P_{n}, n \geq 0$, are the same for $\mu$ and $\nu$. It follows that the operators $a^{-}(j), a^{0}(j)$ and $a^{+}(j)$ are the same for $\mu$ and $\nu$. Hence $A_{\mu}^{0}=A_{\nu}^{0}$ and $A_{\mu}^{-,+}=A_{\nu}^{-,+}$.

$(\Leftarrow)$ Let $\mu$ and $\nu$ be two probability measures on $\mathbb{R}^{d}$, having finite moments of any order, such that $A_{\mu}^{0}=A_{\nu}^{0}$ and $A_{\mu}^{-,+}=A_{\nu}^{-,+}$. Since the equality of two matrices means the equality of their corresponding entries, it follows that for any $i, j, k \in\{1,2, \ldots, d\}$, we have $a_{\mu}^{0}(i)=a_{\nu}^{0}(i)$ and $\left[a_{\mu}^{-}(j), a_{\mu}^{+}(k)\right]=\left[a_{\nu}^{-}(j), a_{\nu}^{+}(k)\right]$, where the subscripts $\mu$ and $\nu$ indicate the probability measure with respect to which a certain operator is calculated.

We will prove by induction on $n$, that for all monomials $m(x)=x_{1}^{i_{1}} \cdots x_{d}^{i_{d}}$ of degree less than or equal to $2 n$, we have $E_{\mu}[m(x)]=E_{\nu}[m(x)]$, where $E_{\mu}$ and $E_{\nu}$ denote the expectations with respect to $\mu$ and $\nu$, respectively.

For $n=0$, there is only one monomial of degree less than or equal to $2 n$, namely the constant polynomial 1 , and we have: $E_{\mu}[1]=E_{\nu}[1]=1$.

Let us assume that all monomials of degree less than or equal to $2 n$ have the same expectation with respect to $\mu$ and $\nu$. Applying the Gram-Schmidt orthogonalization procedure to the set of all monomials of degree less than or equal to $n$, we obtain the same orthonormal basis $\left\{e_{i_{0}}^{0}\right\}_{i_{0} \in I_{0}} \cup\left\{e_{i_{1}}^{1}\right\}_{i_{1} \in I_{1}} \cup \cdots \cup\left\{e_{i_{n}}^{n}\right\}_{i_{n} \in I_{n}}$ for $\mathcal{P}_{n}$, with respect to both probability measures $\mu$ and $\nu$, where for each $0 \leq r \leq n,\left\{e_{i_{r}}^{r}\right\}_{i_{r} \in I_{r}}$ is an orthonormal basis for $G_{r}$. We denote by $\langle,\rangle_{\mu}$ and $\langle,\rangle_{\nu}$ the inner product with respect to $\mu$ and $\nu$, respectively.

Let $m(x)$ be a monomial of degree $2 n+1$. We have $m(x)=x_{j} u(x) v(x)$, for some $j \in\{1,2, \ldots, d\}$ and $u(x)$ and $v(x)$ monomials of degree equal to $n$. Since $u(x) \in \mathcal{P}_{n}$ and $v(x) \in \mathcal{P}_{n}$, we can write:

$$
u(x)=\sum_{i \in I_{n}} \alpha_{i} e_{i}^{n}+\sum_{0 \leq r \leq n-1} \sum_{s \in I_{r}} \beta_{r, s} e_{s}^{r}
$$

and

$$
v(x)=\sum_{i \in I_{n}} \gamma_{i} e_{i}^{n}+\sum_{0 \leq r \leq n-1} \sum_{s \in I_{r}} \delta_{r, s} e_{s}^{r},
$$

where for all $i \in I_{n}, 0 \leq r \leq n-1$, and $s \in I_{r}, \alpha_{i}, \beta_{r, s}, \gamma_{i}$, and $\delta_{r, s}$ are real numbers. 
As $a_{\mu}^{0}(j) e_{i}^{n}=a_{\nu}^{0}(j) e_{i}^{n}$, we obtain:

$$
\sum_{l \in I_{n}}\left\langle x_{j} e_{i}^{n}, e_{l}^{n}\right\rangle_{\mu} e_{l}^{n}=\sum_{l \in I_{n}}\left\langle x_{j} e_{i}^{n}, e_{l}^{n}\right\rangle_{\nu} e_{l}^{n} .
$$

Since the vectors $\left\{e_{l}^{n}\right\}_{l \in I_{n}}$ are linearly independent, we obtain: $\left\langle x_{j} e_{i}^{n}, e_{l}^{n}\right\rangle_{\mu}=$ $\left\langle x_{j} e_{i}^{n}, e_{l}^{n}\right\rangle_{\nu}$, or equivalently:

$$
E_{\mu}\left[x_{j} e_{i}^{n} e_{l}^{n}\right]=E_{\nu}\left[x_{j} e_{i}^{n} e_{l}^{n}\right],
$$

for all $i, l \in I_{n}$.

For all $i \in I_{n}, 0 \leq r \leq n-1$, and $s \in I_{r}$, the degree of the polynomial $x_{j} e_{i}^{n} e_{s}^{r}$ is less than or equal to $2 n$. Thus, according to our induction hypothesis, we have:

$$
E_{\mu}\left[x_{j} e_{i}^{n} e_{s}^{r}\right]=E_{\nu}\left[x_{j} e_{i}^{n} e_{s}^{r}\right] .
$$

For the same reason, for all $0 \leq r_{1} \leq n-1, s_{1} \in I_{r_{1}}, 0 \leq r_{2} \leq n-1$, and $s_{2} \in I_{r_{2}}$, we have:

$$
E_{\mu}\left[x_{j} e_{s_{1}}^{r_{1}} e_{s_{2}}^{r_{2}}\right]=E_{\nu}\left[x_{j} e_{s_{1}}^{r_{1}} e_{s_{2}}^{r_{2}}\right]
$$

Combining all these equalities we get:

$$
\begin{aligned}
E_{\mu}[m(x)]= & E_{\mu}\left[x_{j}\left(\sum_{i \in I_{n}} \alpha_{i} e_{i}^{n}+\sum_{0 \leq r \leq n-1} \sum_{s \in I_{r}} \beta_{r, s} e_{s}^{r}\right)\right. \\
& \left.\times\left(\sum_{i \in I_{n}} \gamma_{i} e_{i}^{n}+\sum_{0 \leq r \leq n-1} \sum_{s \in I_{r}} \delta_{r, s} e_{s}^{r}\right)\right] \\
= & \sum_{i \in I_{n}} \sum_{l \in I_{n}} \alpha_{i} \gamma_{l} E_{\mu}\left[x_{j} e_{i}^{n} e_{l}^{n}\right] \\
& +\sum_{i \in I_{n}} \sum_{0 \leq r \leq n-1} \sum_{s \in I_{r}} \alpha_{i} \delta_{r, s} E_{\mu}\left[x_{j} e_{i}^{n} e_{s}^{r}\right] \\
& +\sum_{i \in I_{n}} \sum_{0 \leq r \leq n-1} \sum_{s \in I_{r}} \gamma_{i} \beta_{r, s} E_{\mu}\left[x_{j} e_{i}^{n} e_{s}^{r}\right] \\
& +\sum_{0 \leq r_{1} \leq n-1} \sum_{s_{1} \in I_{r_{1}}} \sum_{0 \leq r_{2} \leq n-1} \sum_{s_{2} \in I_{r_{2}}} \beta_{r_{1}, s_{1}} \delta_{r_{2}, s_{2}} E_{\mu}\left[x_{j} e_{s_{1}}^{r_{1}} e_{s_{2}}^{r_{2}}\right] \\
= & E_{\nu}[m(x)] .
\end{aligned}
$$

Let $m(x)$ be a monomial of degree $2 n+2$. There exist $j, k \in\{1,2, \ldots, d\}$ and $u(x)$ and $v(x)$ monomials of degree $n$, such that $m(x)=\left(x_{j} u(x)\right)\left(x_{k} v(x)\right)$. Writing, as before,

$$
u(x)=\sum_{i \in I_{n}} \alpha_{i} e_{i}^{n}+\sum_{0 \leq r \leq n-1} \sum_{s \in I_{r}} \beta_{r, s} e_{s}^{r}
$$


and

$$
v(x)=\sum_{i \in I_{n}} \gamma_{i} e_{i}^{n}+\sum_{0 \leq r \leq n-1} \sum_{s \in I_{r}} \delta_{r, s} e_{s}^{r},
$$

showing that $E_{\mu}[m(x)]=E_{\nu}[m(x)]$ reduces to proving that, for all $p, q \in I_{n}$, we have:

$$
E_{\mu}\left[\left(x_{j} e_{p}^{n}\right)\left(x_{k} e_{q}^{n}\right)\right]=E_{\nu}\left[\left(x_{j} e_{p}^{n}\right)\left(x_{k} e_{q}^{n}\right)\right] .
$$

All the other terms are expectations of polynomials of degree less than or equal to $2 n+1$, which are equal with respect to $\mu$ and $\nu$.

Let $p, q \in I_{n}$ be fixed. Let $\left\{g_{s}\right\}_{s \in S}$ be an orthonormal basis of the space $G_{n+1, \mu}:=\mathcal{P}_{n+1} \ominus_{\mu} \mathcal{P}_{n}$ and $\left\{h_{t}\right\}_{t \in T}$ an orthonormal basis of the space $G_{n+1, \nu}:=$ $\mathcal{P}_{n+1} \ominus_{\nu} \mathcal{P}_{n}$. Here, the probability measures are written as subscripts, to indicate that the orthogonal complement is taken with respect to them. As

$$
\left\langle\left[a_{\mu}^{-}(k), a_{\mu}^{+}(j)\right] e_{p}^{n}, e_{q}^{n}\right\rangle_{\mu}=\left\langle\left[a_{\nu}^{-}(k), a_{\nu}^{+}(j)\right] e_{p}^{n}, e_{q}^{n}\right\rangle_{\nu},
$$

we have:

$$
\begin{aligned}
\sum_{s \in S}\left\langle x_{j} e_{p}^{n}, g_{s}\right\rangle_{\mu}\left\langle g_{s}, x_{k} e_{q}^{n}\right\rangle_{\mu}-\sum_{r \in I_{n-1}}\left\langle x_{k} e_{p}^{n}, e_{r}^{n-1}\right\rangle_{\mu}\left\langle e_{r}^{n-1}, x_{j} e_{q}^{n}\right\rangle_{\mu} \\
=\sum_{t \in T}\left\langle x_{j} e_{p}^{n}, h_{t}\right\rangle_{\nu}\left\langle h_{t}, x_{k} e_{q}^{n}\right\rangle_{\nu}-\sum_{r \in I_{n-1}}\left\langle x_{k} e_{p}^{n}, e_{r}^{n-1}\right\rangle_{\nu}\left\langle e_{r}^{n-1}, x_{j} e_{q}^{n}\right\rangle_{\nu} .
\end{aligned}
$$

For all $r \in I_{n-1}$, we have:

$$
\left\langle x_{k} e_{p}^{n}, e_{r}^{n-1}\right\rangle_{\mu}\left\langle e_{r}^{n-1}, x_{j} e_{q}^{n}\right\rangle_{\mu}=\left\langle x_{k} e_{p}^{n}, e_{r}^{n-1}\right\rangle_{\nu}\left\langle e_{r}^{n-1}, x_{j} e_{q}^{n}\right\rangle_{\nu},
$$

because each factor is an expectation of a polynomial of degree at most $2 n$. Thus we obtain:

$$
\sum_{s \in S}\left\langle x_{j} e_{p}^{n}, g_{s}\right\rangle_{\mu}\left\langle g_{s}, x_{k} e_{q}^{n}\right\rangle_{\mu}=\sum_{t \in T}\left\langle x_{j} e_{p}^{n}, h_{t}\right\rangle_{\nu}\left\langle h_{t}, x_{k} e_{q}^{n}\right\rangle_{\nu}
$$

Since $x_{j} e_{p}^{n}$ and $x_{k} e_{q}^{n}$ belong to $G_{n+1, \mu} \oplus G_{n} \oplus G_{n-1}$ for which $\left\{g_{s}\right\}_{s \in S} \cup\left\{e_{l}^{n}\right\}_{l \in I_{n}} \cup$ $\left\{e_{r}^{n-1}\right\}_{r \in I_{n-1}}$ is an orthonormal basis, we conclude that:

$$
\begin{aligned}
\left\langle x_{j} e_{p}^{n}, x_{k} e_{q}^{n}\right\rangle_{\mu}= & \sum_{s \in S}\left\langle x_{j} e_{p}^{n}, g_{s}\right\rangle_{\mu}\left\langle g_{s}, x_{k} e_{q}^{n}\right\rangle_{\mu} \\
& +\sum_{l \in I_{n}}\left\langle x_{j} e_{p}^{n}, e_{l}^{n}\right\rangle_{\mu}\left\langle e_{l}^{n}, x_{k} e_{q}^{n}\right\rangle_{\mu} \\
& +\sum_{r \in I_{n-1}}\left\langle x_{j} e_{p}^{n}, e_{r}^{n-1}\right\rangle_{\mu}\left\langle e_{r}^{n-1}, x_{k} e_{q}^{n}\right\rangle_{\mu}
\end{aligned}
$$

Similarly, we have: 


$$
\begin{aligned}
\left\langle x_{j} e_{p}^{n}, x_{k} e_{q}^{n}\right\rangle_{\nu}= & \sum_{t \in T}\left\langle x_{j} e_{p}^{n}, h_{t}\right\rangle_{\nu}\left\langle h_{t}, x_{k} e_{q}^{n}\right\rangle_{\nu} \\
& +\sum_{l \in I_{n}}\left\langle x_{j} e_{p}^{n}, e_{l}^{n}\right\rangle_{\nu}\left\langle e_{l}^{n}, x_{k} e_{q}^{n}\right\rangle_{\nu} \\
& +\sum_{r \in I_{n-1}}\left\langle x_{j} e_{p}^{n}, e_{r}^{n-1}\right\rangle_{\nu}\left\langle e_{r}^{n-1}, x_{k} e_{q}^{n}\right\rangle_{\nu}
\end{aligned}
$$

We also have:

$$
\begin{aligned}
\sum_{l \in I_{n}}\left\langle x_{j} e_{p}^{n}, e_{l}^{n}\right\rangle_{\mu}\left\langle e_{l}^{n}, x_{k} e_{q}^{n}\right\rangle_{\mu} & \\
& +\sum_{r \in I_{n-1}}\left\langle x_{j} e_{p}^{n}, e_{r}^{n-1}\right\rangle_{\mu}\left\langle e_{r}^{n-1}, x_{k} e_{q}^{n}\right\rangle_{\mu} \\
= & \sum_{l \in I_{n}}\left\langle x_{j} e_{p}^{n}, e_{l}^{n}\right\rangle_{\nu}\left\langle e_{l}^{n}, x_{k} e_{q}^{n}\right\rangle_{\nu} \\
& +\sum_{r \in I_{n-1}}\left\langle x_{j} e_{p}^{n}, e_{r}^{n-1}\right\rangle_{\nu}\left\langle e_{r}^{n-1}, x_{k} e_{q}^{n}\right\rangle_{\nu},
\end{aligned}
$$

since these are sums of products of expectations of polynomials of degree less than or equal to $2 n+1$.

From (3.1)-(3.4) we conclude that:

$$
E_{\mu}\left[\left(x_{j} e_{p}^{n}\right)\left(x_{k} e_{q}^{n}\right)\right]=E_{\nu}\left[\left(x_{j} e_{p}^{n}\right)\left(x_{k} e_{q}^{n}\right)\right] .
$$

The proof is now complete.

As a corollary of this theorem we obtain the following theorem:

Theorem 3.3. The standard Gaussian probability measure on $\mathbb{R}^{d}$, i.e. the probability measure given by the density $g(x)=(2 \pi)^{-n / 2} e^{-|x|^{2} / 2}$, is the only probability measure on $\mathbb{R}^{d}$ for which $A_{\mu}^{-,+}=I_{d}$, where $I_{d}$ is the $d \times d$ operatorial identity matrix, and $A_{\mu}^{0}=0_{d}$, where $0_{d}$ is the $d \times 1$ operatorial zero matrix.

From the Fundamental Theorem, it follows that if a probability measure $\nu$ has the same preservation operators and commutators between the annihilation and creation operators as the standard Gaussian probability measure $\mu$, on $\mathbb{R}^{d}$, then $\nu$ must be moment equal to $\mu$. On the other hand, it is known that a Gaussian is uniquely determined, as a probability measure on $\mathbb{R}^{d}$, by its moments and thus, $\nu=\mu$ (i.e. $\nu(B)=\mu(B)$ for any Borel subset of $\left.\mathbb{R}^{d}\right)$.

The standard Gaussian probability measure on $\mathbb{R}^{d}$ and the $d$-fold product of the Poisson probability measure with mean $\lambda=1$, on $\mathbb{R}$, have the same $A^{-,+}$matrix (see the tables at the end of this paper), even though they are not moment-equal. Thus the matrix $A^{-,+}$alone does not determine uniquely the moments of a probability 
measure. On the other hand, all the polynomially symmetric probability measures have the same $A^{0}=0$ matrix. Thus the matrix $A^{0}$ alone does not determine uniquely the moments of a probability measure either. These facts show that we need both matrices $A^{-,+}$and $A^{0}$, in order to determine uniquely a probability measure from the point of view of moments.

As the Fundamental Theorem involves the operators $a^{0}$ and $\left[a^{-}, a^{+}\right]$, we call each theorem, that characterizes a property of a probability measure in terms of the preservation and commutators between the annihilation and creation operators, a fundamental theorem. In Ref. 2 it was shown that a probability measure on $\mathbb{R}^{d}$, having finite moments of any order, is polynomially symmetric (i.e. all moments of odd degree vanish) if and only if all the preservation operators $a^{0}(j), 1 \leq j \leq d$, are 0 . This is a fundamental theorem. Another theorem from Ref. 2 says that a probability measure on $\mathbb{R}^{d}$, having finite moments of any order, is polynomially factorizable (i.e.

$$
E\left[x_{1}^{i_{1}} x_{2}^{i_{2}} \cdots x_{d}^{i_{d}}\right]=E\left[x_{1}^{i_{1}}\right] E\left[x_{2}^{i_{2}}\right] \cdots E\left[x_{d}^{i_{d}}\right],
$$

for all non-negative integers $i_{1}, i_{2}, \ldots, i_{d}$ ) if and only if, for all $j \neq k$, any operator from the set $\left\{a^{-}(j), a^{0}(j), a^{+}(j)\right\}$ commutes with any operator from the set $\left\{a^{-}(k), a^{0}(i), a^{+}(k)\right\}$. When we say that these operators commute, their domain is understood to be $\mathcal{P}$. This theorem is not fundamental, since it involves the commutators $\left[a^{-}(j), a^{0}(k)\right]$ (which must be zero, for all $j \neq k$ ) and these commutators do not appear in the Fundamental Theorem. On the other hand, the same theorem can be restated as: a probability measure is polynomially factorizable if and only if, for all $j \neq k, a^{0}(j) X_{k}=X_{k} a^{0}(j)$. Written in this way, the theorem is fundamental since it involves the preservation operators $a^{0}$ and the multiplication operators $X$. The multiplication operator $X_{j}$, by the variable $x_{j}$, for $1 \leq j \leq d$, defined on the space $V$, of all polynomial functions of $d$ variables, exists independently of the measure $\mu$, being defined in a pure algebraic way.

We would like to make one more comment. All characterization theorems, that we get in this theory of interacting Fock spaces, in terms of the creation, preservation, and annihilation operators have to contain the adverb "polynomially". Thus, for example, we can characterize the polynomially symmetric probability measures, but not the symmetric ones, and we can also characterize the polynomially factorizable probability measures, but not the factorizable (product) probability measures. In Ref. 2 there is an example of a polynomially symmetric probability measure that is not symmetric and another example of a polynomially factorizable measure that is not factorizable. The fact that we always have to use the word "polynomially" is due to the fact that $a^{-}, a^{0}$ and $a^{+}$are defined in terms of the moments of a probability measure. From the operators $a^{-}, a^{0}$ and $a^{+}$we can recover only the moments of a probability measure. For the probability measures $\mu$, that are not uniquely characterized by their moments, it is not possible to recover the numbers $\mu(B)$, for all Borel sets $B$. 
Despite this fact, it is worth mentioning the following observations, which were suggested to us by Prof. B. Hall. Before presenting them we would like to thank Prof. Hall for his kind advise.

Lemma 3.4. Every polynomially symmetric probability measure is moment equal to a symmetric probability measure.

Proof. Let $\mu$ be a polynomially symmetric probability measure. This means $E\left[x_{1}^{i_{1}} \cdots x_{d}^{i_{d}}\right]=0$, for any monomial $x_{1}^{i_{1}} \cdots x_{d}^{i_{d}}$ of odd degree (i.e. $i_{1}+\cdots+i_{d}$ is odd). Let $\nu$ be the symmetrization of $\mu$. That means for any Borel subset $B$ of $\mathbb{R}^{d}$, we define:

$$
\nu(B):=\frac{1}{2} \mu(B)+\frac{1}{2} \mu(-B),
$$

where $-B:=\{-x \mid x \in B\}$. Then $\nu$ is a symmetric probability measure (i.e. $\nu(-B)=\nu(B)$, for any Borel subset $B$ of $\mathbb{R}^{d}$ ) and it is very easy to see that $\nu$ has the same moments as $\mu$.

Before presenting the next lemma, we would like to recall the following theorem regarding the classical problem of moments.

Theorem 3.5. Let $\left\{m_{i_{1}, i_{2}, \ldots, i_{d}}\right\}_{\left(i_{1}, i_{2}, \ldots, i_{d}\right) \in(\mathbb{N} \cup\{0\})^{d}}$ be a sequence of real numbers. There exists a measure $\mu$ on $\mathbb{R}^{d}$ such that, for all $\left(i_{1}, i_{2}, \ldots, i_{d}\right) \in(\mathbb{N} \cup\{0\})^{d}$, $\int_{\mathbb{R}^{d}} x_{1}^{i_{1}} x_{2}^{i_{2}} \cdots x_{d}^{i_{d}} \mu\left(d x_{1} d x_{2} \cdots d x_{d}\right)=m_{i_{1}, i_{2}, \ldots, i_{d}}$ if and only if for all polynomial functions with real coefficients,

$$
P\left(x_{1}, x_{2}, \ldots, x_{d}\right)=\sum_{k_{1}, k_{2}, \ldots, k_{d}} a_{k_{1}, k_{2}, \ldots, k_{d}} x_{1}^{k_{1}} x_{2}^{k_{2}} \cdots x_{d}^{k_{d}},
$$

such that, for all $\left(x_{1}, x_{2}, \ldots, x_{d}\right) \in \mathbb{R}^{d}, P\left(x_{1}, x_{2}, \ldots, x_{d}\right) \geq 0$, we have $P(m) \geq 0$, where

$$
P(m)=\sum_{k_{1}, k_{2}, \ldots, k_{d}} a_{k_{1}, k_{2}, \ldots, k_{d}} m_{k_{1}, k_{2}, \ldots, k_{d}} .
$$

See Ref. 9 for a proof. The measure from this theorem is a probability measure if and only if $\int_{\mathbb{R}^{d}} 1 \mu(d x)=1$ which means $m_{0,0, \ldots, 0}=1$.

Lemma 3.6. Every polynomially factorizable probability measure is moment equal to a product probability measure.

Proof. Let $\mu$ be a polynomially factorizable probability measure on $\mathbb{R}^{d}$. This means, for any monomial $x_{1}^{i_{1}} x_{2}^{i_{2}} \cdots x_{d}^{i_{d}}$, we have:

$$
E_{\mu}\left[x_{1}^{i_{1}} x_{2}^{i_{2}} \cdots x_{d}^{i_{d}}\right]=E_{\mu}\left[x_{1}^{i_{1}}\right] E_{\mu}\left[x_{2}^{i_{2}}\right] \cdots E_{\mu}\left[x_{d}^{i_{d}}\right],
$$

where $E_{\mu}[\cdot]$ denotes the expectation with respect to $\mu$. For any non-negative integers $i_{1}, i_{2}, \ldots, i_{d}$, we define the numbers:

$$
m_{i_{1}, i_{2}, \ldots, i_{d}}:=E_{\mu}\left[x_{1}^{i_{1}} x_{2}^{i_{2}} \cdots x_{d}^{i_{d}}\right] .
$$


Using the direct implication of Theorem 3.5, we conclude that, for any polynomial $P\left(x_{1}, x_{2}, \ldots, x_{d}\right)$, of $d$ variables, such that $P\left(x_{1}, x_{2}, \ldots, x_{d}\right) \geq 0$, for all $\left(x_{1}\right.$, $\left.x_{2}, \ldots, x_{d}\right) \in \mathbb{R}^{d}$, we have $P(m) \geq 0$, where $P(m)$ is defined as in the text of that theorem.

For any $i \in\{1,2, \ldots, d\}$ and any non-negative integer $\alpha$, we define

$$
m_{\alpha}^{(i)}:=m_{0, \ldots, 0, \alpha, 0, \ldots, 0}
$$

where $\alpha$ is placed on the $i$ th position of the subscript vector $(0, \ldots, 0, \alpha, 0, \ldots, 0)$. Here " $(i)$ " denotes a superscript and not a power. For any polynomial $P(x)$, of only one variable $x$, such that $P(x) \geq 0$, for all $x \in \mathbb{R}$, we may define the polynomial $P_{i}\left(x_{1}, \ldots, x_{d}\right)$, of $d$ variables, by the formula:

$$
P_{i}\left(x_{1}, \ldots, x_{d}\right):=P\left(x_{i}\right) .
$$

Since $P(x) \geq 0$, for all $x \in \mathbb{R}$, we have $P_{i}\left(x_{1}, \ldots, x_{d}\right) \geq 0$, for all $\left(x_{1}, \ldots, x_{d}\right) \in$ $\mathbb{R}^{d}$. This implies that $P\left(m^{(i)}\right)=P_{i}(m) \geq 0$, where $m^{(i)}$ denotes the sequence of real numbers $m_{0}^{(i)}, m_{1}^{(i)}, m_{2}^{(i)}, \ldots$ Thus, according to the converse implication of Theorem 3.5, in the particular case $d=1$, there exists a measure $\mu_{i}$ on $\mathbb{R}$, such that for all non-negative integers $\alpha$, we have:

$$
\int_{\mathbb{R}} x^{\alpha} \mu(d x)=m_{\alpha}^{(i)} .
$$

Since $m_{0, \ldots, 0}=1$, it follows that $m_{0}^{(i)}=1$, and thus $\mu_{i}$ is a probability measure on $\mathbb{R}$.

Let $\nu=\mu_{1} \otimes \mu_{2} \otimes \cdots \otimes \mu_{d}$ be the product measure of $\mu_{1}, \mu_{2}, \ldots, \mu_{d}$. Since $\mu$ is polynomially factorizable, for any monomial $x_{1}^{i_{1}} x_{2}^{i_{2}} \cdots x_{d}^{i_{d}}$, we have:

$$
\begin{aligned}
E_{\mu}\left[x_{1}^{i_{1}} x_{2}^{i_{2}} \cdots x_{d}^{i_{d}}\right] & =E_{\mu}\left[x_{1}^{i_{1}}\right] E_{\mu}\left[x_{2}^{i_{2}}\right] \cdots E_{\mu}\left[x_{d}^{i_{d}}\right] \\
& =m_{i_{1}}^{(1)} m_{i_{2}}^{(2)} \cdots m_{i_{d}}^{(d)} \\
& =E_{\mu_{1}}\left[x_{1}^{i_{1}}\right] E_{\mu_{2}}\left[x_{2}^{i_{2}}\right] \cdots E_{\mu_{d}}\left[x_{d}^{i_{d}}\right] \\
& =E_{\mu_{1} \otimes \mu_{2} \otimes \cdots \otimes \mu_{d}}\left[x_{1}^{i_{1}} x_{2}^{i_{2}} \cdots x_{d}^{i_{d}}\right] \\
& =E_{\nu}\left[x_{1}^{i_{1}} x_{2}^{i_{2}} \cdots x_{d}^{i_{d}}\right] .
\end{aligned}
$$

Thus $\mu$ is moment equal to $\nu$ which is a product measure, even though $\mu$ is not necessary of a product measure.

The above two lemmas show that, modulo the equivalence relation $\stackrel{m}{=}$, the notions of "polynomially symmetry" and "polynomially factorizability" are the same as the notions of "symmetry" and "factorizability", respectively.

\section{Existence Problem}

In this section we will discuss the following problem. Let $\left\{a_{i, j}\right\}_{1 \leq i, j \leq d}$ and $\left\{b_{k}\right\}_{1 \leq k \leq d}$ be two families of linear maps defined on the vector space $V$ of all 
polynomial functions, in $d$ variables, with values in the same space $V$. What conditions must these two families of operators satisfy to ensure the existence of a probability measure $\mu$ on $\mathbb{R}^{d}$, having finite moments of any order, such that for all $i, j, k \in\{1,2, \ldots, d\},\left[a_{\mu}^{-}(i), a_{\mu}^{+}(j)\right]=a_{i, j}$ and $a_{\mu}^{0}(k)=b_{k}$ ?

We will find some necessary conditions.

For all $n \geq 0$, let $V_{n}$ be the vector space of all polynomial functions, in $d$ variables, of degree less than or equal to $n$. It is clear that, if such a probability measure $\mu$ exists, then for all $n \geq 0, V_{n}$ is invariant under the action of all the operators $a_{i, j}$ and $b_{k}$. That means, for all $n \geq 0$ and $i, j, k \in\{1,2, \ldots, d\}, a_{i, j} V_{n} \subset$ $V_{n}$ and $b_{k} V_{n} \subset V_{n}$.

\subsection{The one-dimensional case $(d=1)$}

We will now present some known results for the case $d=1$. If $d=1$ and $\mu$ is a probability measure on the Borel subsets of $\mathbb{R}$, having finite moments of any order, then we have only one variable $x_{1}$ denoted by $x$ and we can simply write $a_{\mu}^{-}, a_{\mu}^{0}$, and $a_{\mu}^{+}$instead of $a_{\mu}^{-}(1), a_{\mu}^{0}(1)$ and $a_{\mu}^{+}(1)$, respectively. Since the algebraic codimension of $V_{n-1}$ into $V_{n}$ is 1 , the dimension of the space $G_{n}$ is less than or equal to 1 , for all $n \geq 0$.

If $\mu$ is a probability measure on $\mathbb{R}$ having finite moments of any order such that the dimension of $G_{n}$ is 1 , for all $n \geq 0$, then we denote by $e_{n}$ a polynomial of norm 1 in $G_{n}$. Thus, $\left\{e_{n}\right\}$ is an orthonormal basis of $G_{n}$. Since $G_{n}$ has dimension 1, there is only one polynomial in $G_{n}$ having the leading coefficient (i.e. the coefficient of $x^{n}$ ) equal to 1 . We denote this polynomial by $P_{n}$, for all $n \geq 0$. We would like to warn the reader, to distinguish between the polynomial $P_{n}$ and the projection operator of the Hilbert space $\mathcal{H}$ onto its closed subspace $G_{n}$, which was also denoted before by $P_{n}$, for all $n \geq 0$. It would have been better to use another notation for the polynomials $P_{n}$, but we have not changed it because this notation appears in many other papers. Of course, $P_{0}=1$ (i.e. the constant polynomial 1). Since $x P_{n} \in G_{n+1} \oplus G_{n} \oplus G_{n-1}$ and $x P_{n}$ is a polynomial of degree $n+1$ having the leading coefficient 1 , there exist two real numbers $\alpha_{n}$ and $\omega_{n}$, such that:

$$
x P_{n}=P_{n+1}+\alpha_{n} P_{n}+\omega_{n} P_{n-1}, \quad \forall n \geq 0 .
$$

In the above relation $P_{-1}=0$ and $\omega_{0}=0$ by convention. The sequences of numbers $\left\{\alpha_{n}\right\}_{n \geq 0}$ and $\left\{\omega_{n}\right\}_{n \geq 1}$ are called the Szegö-Jacobi parameters of $\mu$.

If the dimension of the space $G_{n}$ is zero, for some positive integer $n$, then denoting by $n_{0}$, the smallest of all such positive integers, we get, $G_{n}=\{0\}$ (the null space), for all $n \geq n_{0}$. In this case, we can define, for all $n \geq n_{0}, \alpha_{n}:=0$, $\omega_{n}:=0$, and $P_{n}:=0$. Then, for $n=n_{0}-1$, the equality (4.1) must be understood in the $\mu$-almost surely sense.

We denote by $\lambda_{n}$ the square of the norm of $P_{n}$ in the space $L^{2}(\mathbb{R}, \mu)$. It is known that $\lambda_{n}=\omega_{1} \omega_{2} \cdots \omega_{n}$, for all $n \geq 1$. From this relation it follows easily that $\omega_{n} \geq 0$, for all $n \geq 1$. Actually it is easy to see that the condition $\omega_{n} \geq 0$, for all $n \geq 1$, is not only necessary, but also almost sufficient because of the following results. 
Theorem 4.1. Let $\left\{\alpha_{n}\right\}_{n \geq 0}$ and $\left\{\omega_{n}\right\}_{n \geq 1}$ be two sequences of real numbers. Then there exists a probability measure $\mu$ on $\mathbb{R}$, having finite support of cardinality $m$, such that the Szegö-Jacobi parameters of $\mu$ are $\left\{\alpha_{n}\right\}_{n \geq 0}$ and $\left\{\omega_{n}\right\}_{n \geq 1}$ if and only if, for all $k \in\{1,2, \ldots, m-1\}, \omega_{k}>0$, for all $k \geq m, \omega_{k}=0$, and for all $k \geq m$, $\alpha_{k}=0$.

Theorem 4.2. Let $\left\{\alpha_{n}\right\}_{n \geq 0}$ and $\left\{\omega_{n}\right\}_{n \geq 1}$ be two sequences of real numbers. Then there exists a probability measure $\mu$ on $\mathbb{R}$, of infinite support, having finite moments of any order, such that the Szegö-Jacobi parameters of $\mu$ are $\left\{\alpha_{n}\right\}_{n \geq 0}$ and $\left\{\omega_{n}\right\}_{n \geq 1}$ if and only if, for all $k \geq 1, \omega_{k}>0$.

The probability measures with compact support can also be characterized in terms of the Szegö-Jacobi parameters, by the following theorem:

Theorem 4.3. Let $\mu$ be a probability measure on $\mathbb{R}$, having finite moments of any order. Let $\left\{\alpha_{n}\right\}_{n \geq 0}$ and $\left\{\omega_{n}\right\}_{n \geq 1}$ be the Szegö-Jacobi parameters of $\mu$. Then $\mu$ has compact support if and only if both sequences $\left\{\alpha_{n}\right\}_{n \geq 0}$ and $\left\{\omega_{n}\right\}_{n \geq 1}$ are bounded.

If $E$ is a finite-dimensional vector space, without any topology, and $T$ is a linear map from $E$ into $E$, then we can define the trace of $T, \operatorname{Tr}(T)$, by fixing an algebraic basis $\left\{e_{i}\right\}_{1 \leq i \leq n}$ of $E$. If $A=\left(a_{i j}\right)_{1 \leq i, j \leq n}$ is the matrix associated to $T$ with respect to the basis $\left\{e_{i}\right\}_{1 \leq i \leq n}$, for both domain and codomain $E$, of $T$, then we define:

$$
\operatorname{Tr}(T)=\sum_{i=1}^{n} a_{i i} .
$$

This definition is known to be independent of the choice of the basis of $E$.

If $\mu$ is a probability measure on $\mathbb{R}$, having finite moments of any order, and $\left\{\alpha_{n}\right\}_{n \geq 0}$ and $\left\{\omega_{n}\right\}_{n \geq 1}$ are its Szegö-Jacobi parameters, then from the recurrence relation (4.1), we can see that $a_{\mu}^{-} P_{n}=\omega_{n} P_{n-1}$ and $a_{\mu}^{+} P_{n}=P_{n+1}$. Thus, we obtain:

$$
\begin{aligned}
{\left[a_{\mu}^{-}, a_{\mu}^{+}\right] P_{n} } & =a_{\mu}^{-} a_{\mu}^{+} P_{n}-a_{\mu}^{+} a_{\mu}^{-} P_{n} \\
& =a_{\mu}^{-} P_{n+1}-a_{\mu}^{+}\left(\omega_{n} P_{n-1}\right) \\
& =\omega_{n+1} P_{n}-\omega_{n} P_{n} \\
& =\left(\omega_{n+1}-\omega_{n}\right) P_{n} .
\end{aligned}
$$

Using the algebraic (in fact orthogonal, but not necessary orthonormal) basis $\left\{P_{0}, P_{1}, \ldots, P_{n}\right\}$ of $V_{n}$, and denoting by $\left.\left[a_{\mu}^{-}, a_{\mu}^{+}\right]\right|_{V_{n}}$ the restriction of $\left[a_{\mu}^{-}, a_{\mu}^{+}\right]$to its invariant subspace $V_{n}$, we have:

$$
\begin{aligned}
\operatorname{Tr}\left(\left.\left[a_{\mu}^{-}, a_{\mu}^{+}\right]\right|_{V_{n}}\right) & =\sum_{k=0}^{n}\left(\omega_{k+1}-\omega_{k}\right) \\
& =\omega_{n+1} .
\end{aligned}
$$


Using the same algebraic basis of $V_{n}$, as before, we can easily see that

$$
\operatorname{Tr}\left(\left.a_{\mu}^{0}\right|_{V_{n}}\right)=\sum_{k=0}^{n} \alpha_{k}
$$

and thus

$$
\alpha_{n}=\operatorname{Tr}\left(\left.a_{\mu}^{0}\right|_{V_{n}}\right)-\operatorname{Tr}\left(\left.a_{\mu}^{0}\right|_{V_{n-1}}\right)
$$

for all $n \geq 0$, where by convention $V_{-1}:=\{0\}$ is the null space.

We can rewrite Theorems 4.1-4.3 as follows:

Theorem 4.4. Let $m$ be a fixed natural number and $a^{0}$ and $a^{-,+}$two linear maps on the space $V$ of all polynomial functions of one variable $x$. We assume that, for all $n \geq 0$, the subspace $V_{n}$, of polynomial functions of degree at most $n$, is invariant under the action of both $a^{0}$ and $a^{-,+}$. There exists a probability measure $\mu$ on $\mathbb{R}$, of finite support of cardinality $m$, such that, for all $n \geq 0, \operatorname{Tr}\left(\left.a^{0}\right|_{V_{n}}\right)=\operatorname{Tr}\left(\left.a_{\mu}^{0}\right|_{V_{n}}\right)$ and $\operatorname{Tr}\left(\left.a^{-,+}\right|_{V_{n}}\right)=\operatorname{Tr}\left(\left.\left[a_{\mu}^{-}, a_{\mu}^{+}\right]\right|_{V_{n}}\right)$ if and only if, for all $k \leq m-2, \operatorname{Tr}\left(\left.a^{-,+}\right|_{V_{k}}\right)>0$, for all $k \geq m-1, \operatorname{Tr}\left(\left.a^{-,+}\right|_{V_{k}}\right)=0$, and for all $k \geq m-1, \operatorname{Tr}\left(\left.a^{0}\right|_{V_{k}}\right)=\operatorname{Tr}\left(\left.a^{0}\right|_{V_{m-1}}\right)$.

Theorem 4.5. Let $a^{0}$ and $a^{-,+}$be two linear maps on the space $V$ of all polynomial functions of one variable $x$. We assume that, for all $n \geq 0$, the subspace $V_{n}$, of polynomial functions of degree at most $n$, is invariant under the action of both $a^{0}$ and $a^{-,+}$. There exists a probability measure $\mu$ on $\mathbb{R}$, of infinite support, having finite moments of any order, such that, for all $n \geq 0, \operatorname{Tr}\left(\left.a^{0}\right|_{V_{n}}\right)=\operatorname{Tr}\left(\left.a_{\mu}^{0}\right|_{V_{n}}\right)$ and $\operatorname{Tr}\left(\left.a^{-,+}\right|_{V_{n}}\right)=\operatorname{Tr}\left(\left.\left[a_{\mu}^{-}, a_{\mu}^{+}\right]\right|_{V_{n}}\right)$ if and only if, for all $k \geq 0, \operatorname{Tr}\left(\left.a^{-,+}\right|_{V_{k}}\right)>0$.

Theorem 4.6. A probability measure $\mu$ on $\mathbb{R}$, has compact support, if and only if it has finite moments of any order and both sequences of real numbers $\left\{\operatorname{Tr}\left(\left.a_{\mu}^{0}\right|_{V_{n}}\right)-\right.$ $\left.\operatorname{Tr}\left(\left.a_{\mu}^{0}\right|_{V_{n-1}}\right)\right\}_{n \geq 1}$ and $\left\{\operatorname{Tr}\left(\left.\left[a_{\mu}^{-}, a_{\mu}^{+}\right]\right|_{V_{n}}\right)\right\}_{n \geq 0}$ are bounded, where $V_{n}$ denotes the space of all polynomial functions, of one variable $x$, of degree at most $n$.

Theorems 4.1 and 4.2, or their equivalent Theorems 4.4 and 4.5, respectively, give us a "satisfactory" answer to the existence problem in the dimension $d=1$ case. The answer is only satisfactory because it does not tell us anything about the non-diagonal entries of the matrices associated to $\left.a^{0}\right|_{V_{n}}$ and $\left.a^{-,+}\right|_{V_{n}}$ with respect to various bases of $V_{n}$, for $n \geq 0$. They give us information only about the eigenvalues of these matrices, which are $\left\{\alpha_{k}\right\}_{k=0}^{n}$ and $\left\{\omega_{k+1}-\omega_{k}\right\}_{k=0}^{n}$, respectively, for all $n \geq 0$, or, equivalently, about their traces. Since this information is enough to recover all the moments of the probability measure $\mu$, perhaps, the existence problem is not well posed and should be reformulated by requiring, not to find all the linear operators which are the preservation and commutators between the annihilation and creation operators of a probability measure, having finite moments of any order, but to find only those characteristics of these operators that are enough to ensure the existence of such a probability measure. 


\subsection{The multidimensional case $(d \geq 2)$}

Let us remember the problem that we are trying to solve. Let $\left\{a_{i, j}\right\}_{1 \leq i, j \leq d}$ and $\left\{b_{k}\right\}_{1 \leq k \leq d}$ be two families of linear maps defined on the vector space $V$ of all polynomial functions, in $d$ variables, with values in the same space $V$. What conditions must these two families of operators satisfy to ensure the existence of a probability measure $\mu$ on $\mathbb{R}^{d}$, having finite moments of any order, such that for all $i, j$, $k \in\{1,2, \ldots, d\},\left[a_{\mu}^{-}(i), a_{\mu}^{+}(j)\right]=a_{i, j}$ and $a_{\mu}^{0}(k)=b_{k}$ ?

At this moment there is no inner product given on $V$ since the probability measure $\mu$ has not been constructed yet. Thus $V$ has only an algebraic structure, being a vector space. It has an increasing sequence of well-defined vector subspaces:

$$
V_{0} \subset V_{1} \subset V_{2} \subset \cdots \subset V
$$

where $V_{n}$ denotes the space of all polynomial functions, of $d$ variables: $x_{1}, x_{2}, \ldots, x_{d}$, of degree at most $n$. All the conditions that we formulate should have a pure algebraic meaning. One of the necessary conditions is that all the spaces $V_{n}, n \geq 0$, be invariant under the action of each of the operators $a_{i, j}, 1 \leq i, j \leq d$, and $b_{k}$, $1 \leq k \leq d$.

Let us assume that there exists a probability measure $\mu$ on the Borel subsets of $\mathbb{R}^{d}$, having finite moments of any order, such that for all $i, j, k \in\{1,2, \ldots, d\}$, $\left[a_{\mu}^{-}(i), a_{\mu}^{+}(j)\right]=a_{i, j}$ and $a_{\mu}^{0}(k)=b_{k}$. Let us also recall the relation $(2.9)$ :

$$
\begin{aligned}
& D_{n+1}^{-}(i) D_{n}^{+}(j)+D_{n}^{0}(i) D_{n}^{0}(j)+D_{n-1}^{+}(i) D_{n}^{-}(j) \\
& \quad=D_{n+1}^{-}(j) D_{n}^{+}(i)+D_{n}^{0}(j) D_{n}^{0}(i)+D_{n-1}^{+}(j) D_{n}^{-}(i)
\end{aligned}
$$

for all $i, j \in\{1,2, \ldots, d\}$ and all $n \geq 0$. This relation is equivalent to

$$
\begin{aligned}
\left(D_{n+1}^{-}(i) D_{n}^{+}(j)-D_{n-1}^{+}(j) D_{n}^{-}(i)\right) & \\
& -\left(D_{n+1}^{-}(j) D_{n}^{+}(i)-D_{n-1}^{+}(i) D_{n}^{-}(j)\right) \\
= & D_{n}^{0}(j) D_{n}^{0}(i)-D_{n}^{0}(i) D_{n}^{0}(j)
\end{aligned}
$$

which means that:

$$
\left[a_{\mu}^{-}(i), a_{\mu}^{+}(j)\right]-\left[a_{\mu}^{-}(j), a_{\mu}^{+}(i)\right]=\left[a_{\mu}^{0}(j), a_{\mu}^{0}(i)\right]
$$

for all $i, j \in\{1,2, \ldots, d\}$. 
Thus, we obtain the necessary condition:

$$
a_{i, j}-a_{j, i}=\left[b_{j}, b_{i}\right],
$$

for all $i, j \in\{1,2, \ldots, d\}$.

Let us recall now the commutation relation (2.7):

$$
D_{n+1}^{+}(i) D_{n}^{+}(j)=D_{n+1}^{+}(j) D_{n}^{+}(i),
$$

for all $i, j \in\{1,2, \ldots, d\}$ and all $n \geq 0$. This is equivalent to

$$
a_{\mu}^{+}(i) a_{\mu}^{+}(j)=a_{\mu}^{+}(j) a_{\mu}^{+}(i),
$$

for all $i, j \in\{1,2, \ldots, d\}$. Taking the adjoint on both sides of this equality, we obtain:

$$
a_{\mu}^{-}(j) a_{\mu}^{-}(i)=a_{\mu}^{-}(i) a_{\mu}^{-}(j),
$$

for all $i, j \in\{1,2, \ldots, d\}$. Using now the relations $X_{i}=a_{\mu}^{-}(i)+a_{\mu}^{0}(i)+a_{\mu}^{+}(i)$, $X_{j}=a_{\mu}^{-}(j)+a_{\mu}^{0}(j)+a_{\mu}^{+}(j),(4.2),(4.4)$ and (4.5), and the commutativity between the multiplication operators $X_{i}$ and $X_{j}$, we obtain:

$$
\begin{aligned}
{\left[a_{\mu}^{0}(i), a_{\mu}^{0}(j)\right] } & =\left[a_{\mu}^{-}(j), a_{\mu}^{+}(i)\right]-\left[a_{\mu}^{-}(i), a_{\mu}^{+}(j)\right] \\
& =\left[a_{\mu}^{-}(j), a_{\mu}^{+}(i)\right]+\left[a_{\mu}^{+}(j), a_{\mu}^{-}(i)\right] \\
& =\left[a_{\mu}^{-}(j)+a_{\mu}^{+}(j), a_{\mu}^{-}(i)+a_{\mu}^{+}(i)\right] \\
& =\left[X_{j}-a_{\mu}^{0}(j), X_{i}-a_{\mu}^{0}(i)\right] \\
& =\left[X_{j}, X_{i}\right]-\left[X_{j}, a_{\mu}^{0}(i)\right]-\left[a_{\mu}^{0}(j), X_{i}\right]+\left[a_{\mu}^{0}(j), a_{\mu}^{0}(i)\right] \\
& =\left[a_{\mu}^{0}(i), X_{j}\right]-\left[a_{\mu}^{0}(j), X_{i}\right]-\left[a_{\mu}^{0}(i), a_{\mu}^{0}(j)\right],
\end{aligned}
$$

for all $i, j \in\{1,2, \ldots, d\}$. This implies:

$$
\left[a_{\mu}^{0}(i), X_{j}\right]-\left[a_{\mu}^{0}(j), X_{i}\right]=2\left[a_{\mu}^{0}(i), a_{\mu}^{0}(j)\right],
$$

for all $i, j \in\{1,2, \ldots, d\}$.

Therefore, we have obtained another necessary condition:

$$
\left[b_{i}, X_{j}\right]-\left[b_{j}, X_{i}\right]=2\left[b_{i}, b_{j}\right],
$$

for all $i, j \in\{1,2, \ldots, d\}$.

We would like to mention again that (4.7) is a well-defined algebraic relation since the multiplication operators $X_{i}$ and $X_{j}$, defined on the space of all polynomial functions $V$ with values in $V$, have a clear algebraic meaning and they do not depend on the existence of any probability measure $\mu$. The domain of all operators: $X_{i}, X_{j}$, $b_{i}$ and $b_{j}$, involved in (4.7), is considered to be $V$.

We present now a necessary condition that extends the requirement that $\omega_{n} \geq 0$, for all $n \geq 1$, from the one-dimensional case $d=1$ to the multidimensional case $d \geq 2$. 
Lemma 4.7. Let $\mu$ be a probability measure on $\mathbb{R}^{d}$, having finite moments of any order. Then, for all $n \geq 0,\left(\operatorname{Tr}\left(\left.\left[a_{\mu}^{-}(i), a_{\mu}^{+}(j)\right]\right|_{V_{n}}\right)\right)_{1 \leq i, j \leq d}$ is a $d \times d$ positive semidefinite matrix.

Proof. For each $n \geq 0$, let $\left\{e_{i}^{n}\right\}_{1 \leq i \leq t_{n}}$ be an orthonormal basis of the space $G_{n}$. Then for any $k \geq 0$ and $z_{1}, z_{2}, \ldots, z_{d} \in \mathbb{C}$, we have:

$$
\begin{aligned}
\sum_{1 \leq i, j \leq d} z_{i} \bar{z}_{j} \sum_{1 \leq u \leq t_{k}}\left\langle\left[a_{\mu}^{-}(i), a_{\mu}^{+}(j)\right] e_{u}^{k}, e_{u}^{k}\right\rangle \\
=\sum_{1 \leq i, j \leq d} z_{i} \bar{z}_{j} \sum_{1 \leq u \leq t_{k}} \sum_{1 \leq v \leq t_{k+1}}\left\langle X_{j} e_{u}^{k}, e_{v}^{k+1}\right\rangle\left\langle X_{i} e_{u}^{k}, e_{v}^{k+1}\right\rangle \\
\quad-\sum_{1 \leq i, j \leq d} z_{i} \bar{z}_{j} \sum_{1 \leq u \leq t_{k}} \sum_{1 \leq w \leq t_{k-1}}\left\langle X_{j} e_{w}^{k-1}, e_{u}^{k}\right\rangle\left\langle X_{i} e_{w}^{k-1}, e_{u}^{k}\right\rangle .
\end{aligned}
$$

Summing up from $k=0$ to $k=n$, we obtain:

$$
\begin{aligned}
& \sum_{1 \leq i, j \leq d} z_{i} \bar{z}_{j} \operatorname{Tr}\left(\left.\left[a_{\mu}^{-}(i), a_{\mu}^{+}(j)\right]\right|_{V_{n}}\right) \\
& \quad=\sum_{1 \leq i, j \leq d} z_{i} \bar{z}_{j} \sum_{1 \leq u \leq t_{n}} \sum_{1 \leq v \leq t_{n+1}}\left\langle X_{j} e_{u}^{n}, e_{v}^{n+1}\right\rangle\left\langle X_{i} e_{u}^{n}, e_{v}^{n+1}\right\rangle \\
& =\sum_{1 \leq i, j \leq d} \sum_{1 \leq u \leq t_{n}} \sum_{1 \leq v \leq t_{n+1}}\left\langle z_{i} X_{i} e_{u}^{n}, e_{v}^{n+1}\right\rangle\left\langle e_{v}^{n+1}, z_{j} X_{j} e_{u}^{n}\right\rangle \\
& =\sum_{1 \leq i, j \leq d} \sum_{1 \leq u \leq t_{n}}\left\langle z_{i} D_{n}^{+}(i) e_{u}^{n}, z_{j} D_{n}^{+}(j) e_{u}^{n}\right\rangle \\
& =\sum_{1 \leq u \leq t_{n}}\left\|\sum_{1 \leq i \leq d} z_{i} D_{n}^{+}(i) e_{u}^{n}\right\|^{2} \\
& =\left\|\sum_{1 \leq i \leq d} z_{i} D_{n}^{+}(i)\right\|_{\mathrm{HS}} \\
& \geq 0
\end{aligned}
$$

where $\|\cdot\|_{\text {HS }}$ denotes the Hilbert-Schmidt norm.

Thus we obtain the following necessary condition: for all $n \geq 0$, the matrix $\left(\operatorname{Tr}\left(\left.a_{i, j}\right|_{V_{n}}\right)\right)_{1 \leq i, j \leq d}$ must be positive semidefinite.

We summarize now all the necessary conditions that we have found so far.

Theorem 4.8. Let $\left\{a_{i, j}\right\}_{1 \leq i, j \leq d}$ and $\left\{b_{k}\right\}_{1 \leq k \leq d}$ be two families of linear maps from the vector space $V$, of all polynomial functions of $d$ variables: $x_{1}, x_{2}, \ldots, x_{d}$, into itself. If there exists a probability measure $\mu$ on $\mathbb{R}^{d}$, having finite moments of any 
order, such that for all $i, j, k \in\{1,2, \ldots, d\},\left[a_{\mu}^{-}(i), a_{\mu}^{+}(j)\right]=a_{i, j}$ and $a_{\mu}^{0}(k)=b_{k}$, then the following conditions hold:

(1) For all $n \geq 0$ and all $i, j, k \in\{1,2, \ldots, d\}, a_{i, j} V_{n} \subset V_{n}$ and $b_{k} V_{n} \subset V_{n}$, where $V_{n}$ denotes the space of all polynomial functions of $d$ variables, of degree at most $n$.

(2) For all $i, j \in\{1,2, \ldots, d\}, a_{i, j}-a_{j, i}=\left[b_{j}, b_{i}\right]$.

(3) For all $i, j \in\{1,2, \ldots, d\},\left[b_{i}, X_{j}\right]-\left[b_{j}, X_{i}\right]=2\left[b_{i}, b_{j}\right]$.

(4) For all $n \geq 0$, the matrix $\left(\operatorname{Tr}\left(\left.a_{i, j}\right|_{V_{n}}\right)\right)_{1 \leq i, j \leq d}$ is positive semidefinite.

Comment. In the one-dimensional case $d=1$ we saw that knowing the SzegöJacobi parameters $\left\{\alpha_{n}\right\}_{n \geq 0}$ and $\left\{\omega_{n}\right\}_{n \geq 1}$, or equivalently knowing all the traces $\left\{\operatorname{Tr}\left(\left.a^{0}\right|_{V_{n}}\right)\right\}_{n \geq 0}$ and $\left\{\operatorname{Tr}\left(\left.\left[a^{-}, a^{+}\right]\right|_{V_{n}}\right)\right\}_{n \geq 0}$, is the same as knowing all the moments of the probability measure $\mu$. In fact the numbers $\left\{\alpha_{n}\right\}_{n \geq 0}$ and $\left\{\omega_{n+1}-\omega_{n}\right\}_{n \geq 0}$ are the eigenvalues of the operators $a^{0}$ and $\left[a^{-}, a^{+}\right]$, respectively, whose domain is considered to be $V$. These eigenvalues are the roots of the characteristic polynomials of the two operators restricted to the spaces $V_{n}, n \geq 0$. The characteristic polynomials can be defined purely algebraically, without introducing any inner product on the spaces $V_{n}, n \geq 0$. The existence theorems are easy to prove for $d=1$, because the codimension of $V_{n-1}$ into $V_{n}$ is 1 , and we have just one variable $x$. Due to this fact, the eigenvalues of $a^{0}$ can be recovered as $\operatorname{Tr}\left(\left.a^{0}\right|_{V_{n}}\right)-\operatorname{Tr}\left(\left.a^{0}\right|_{V_{n-1}}\right)$, for $n \geq 0$.

The existence problem for $d \geq 2$, which we were not able to solve, is much more difficult perhaps because the codimension of $V_{n-1}$ into $V_{n}$ is $\left(\begin{array}{c}n+d-1 \\ d-1\end{array}\right)$. We have formulated a necessary condition involving the traces. The trace is only the second coefficient (after the dominant coefficient which is 1) of the characteristic polynomial of a matrix, and there may be some necessary conditions about the other coefficients of the characteristic polynomial of the operators $\left.\left[a^{-}, a^{+}\right]\right|_{V_{n}}, n \geq 0$, or equivalently about the eigenvalues of these operators. Knowing only the traces is not enough to find all the eigenvalues.

Another very important difference between the case $d=1$ and $d \geq 2$, is given by the commutativity conditions (4.3) and (4.7) which appear only in the case $d \geq 2$. If the condition about the matrix of traces being positive semidefinite refers to the eigenvalues of the operators $\left[a^{-}(i), a^{+}(j)\right]$, for $1 \leq i, j \leq d$, the commutativity conditions (4.3) and (4.7) may refer to the eigenvectors of some matrices. For example, it is known that two self-adjoint matrices commute if and only if they can be diagonalized in the same basis.

In conclusion, even though our results, in the case $d \geq 2$, are incomplete, they open some small doors, into the unknown, which may lead not only to a complete solution of the existence problem, but also to a good definition of the Szegö-Jacobi parameters, in the multidimensional case, that naturally extends the one from the $d=1$ case. 


\section{The Operators $a^{0}$ and $\left[a^{-}, a^{+}\right]$for Some Classical One-Dimensional Distributions}

We present below the formulas of the Szegö-Jacobi parameters and the commutator $\left[a^{-}, a^{+}\right]$for some classic one-dimensional distributions. See also Refs. $6-8$.

Table 1.

\begin{tabular}{|c|c|}
\hline Measure & Polynomial $P_{n}$ \\
\hline $\begin{array}{l}\text { Gaussian } \\
N\left(0, \sigma^{2}\right)\end{array}$ & $\begin{array}{l}\text { Hermite } \\
H_{n}\left(x ; \sigma^{2}\right)=\left(-\sigma^{2}\right)^{n} e^{x^{2} / 2 \sigma^{2}} \partial_{x}^{n} e^{-x^{2} / 2 \sigma^{2}}\end{array}$ \\
\hline $\begin{array}{l}\text { Poisson } \\
\text { Poi }(a)\end{array}$ & $\begin{array}{l}\text { Charlier } \\
C_{n}(x ; a)=(-1)^{n} a^{-x} \Gamma(x+1) \Delta_{x+}^{n}\left[\frac{a^{x}}{\Gamma(x-n+1)}\right. \\
\Delta_{x+} f(x)=f(x+1)-f(x)\end{array}$ \\
\hline $\begin{array}{ll}\operatorname{Gamma} \Gamma(\alpha), & (\alpha>0) \\
\frac{1}{\Gamma(\alpha)} x^{\alpha-1} e^{-x}, & x>0\end{array}$ & $\begin{array}{l}\text { Laguerre } \\
\mathcal{L}_{n}^{(\alpha-1)}(x)=(-1)^{n} x^{-\alpha+1} e^{x} \partial_{x}^{n}\left[x^{n+\alpha-1} e^{-x}\right]\end{array}$ \\
\hline Uniform on $[-1,1]$ & $\begin{array}{l}\text { Legendre } \\
\tilde{L}_{n}(x)=\frac{1}{2^{n}(2 n-1) ! !} \partial_{x}^{n}\left[\left(x^{2}-1\right)^{n}\right]\end{array}$ \\
\hline $\begin{array}{l}\text { Arcsine } \\
\frac{1}{\pi \sqrt{1-x^{2}}},|x|<1\end{array}$ & $\begin{array}{l}\text { Chebyshev (first kind) } \\
\tilde{T}_{0}(x)=1 \\
\tilde{T}_{n}(x)=\frac{1}{2^{n-1}} \cos \left(n \cos ^{-1} x\right), n \geq 1\end{array}$ \\
\hline $\begin{array}{l}\text { Semicircle } \\
\frac{2}{\pi} \sqrt{1-x^{2}},|x|<1\end{array}$ & $\begin{array}{l}\text { Chebyshev (second kind) } \\
\tilde{U}_{n}(x)=\frac{1}{2^{n}} \frac{\sin \left[(n+1) \cos ^{-1} x\right]}{\sin \left(\cos ^{-1} x\right)}\end{array}$ \\
\hline $\begin{array}{r}\frac{1}{\sqrt{\pi}} \frac{\Gamma(\beta+1)}{\Gamma\left(\beta+\frac{1}{2}\right)}\left(1-x^{2}\right)^{\beta-\frac{1}{2}},|x|<1 \\
\beta>-\frac{1}{2}, \beta \neq 0,1\end{array}$ & $\begin{array}{l}\text { Gegenbauer } \\
\tilde{G}_{n}^{(\beta)}=C_{n}^{(\beta)}\left(1-x^{2}\right)^{\frac{1}{2}-\beta} u(x), \\
u(x)=\partial_{x}^{n}\left[\left(1-x^{2}\right)^{n+\beta-\frac{1}{2}}\right] \\
C_{n}^{(\beta)}=\frac{(-1)^{n} 2^{n} \Gamma(2 \beta+n)}{\Gamma(2 \beta+2 n)}\end{array}$ \\
\hline $\begin{array}{l}\text { Negative binomial } \\
r>0,0<p<1 \\
P(X=x)=p^{r}\left(\begin{array}{c}-r \\
x\end{array}\right)(-1)^{x}(1-p)^{x}, \\
x \in \mathbb{N} \cup\{0\}\end{array}$ & 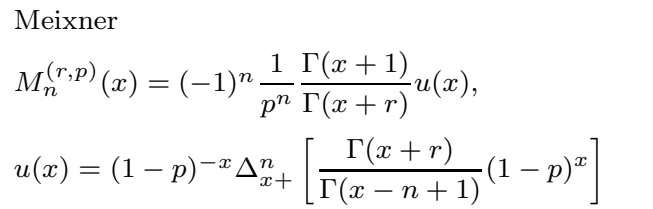 \\
\hline
\end{tabular}


Table 2 .

\begin{tabular}{|c|c|}
\hline Measure & Szegö-Jacobi parameters \\
\hline $\begin{array}{l}\text { Gaussian } \\
N\left(0, \sigma^{2}\right)\end{array}$ & $\begin{aligned} \alpha_{n} & =0 \\
\omega_{n} & =\sigma^{2} n \\
\left(\lambda_{n}\right. & \left.=\sigma^{2 n} n !\right)\end{aligned}$ \\
\hline $\begin{array}{l}\text { Poisson } \\
\text { Poi }(a)\end{array}$ & $\begin{aligned} \alpha_{n} & =n+a \\
\omega_{n} & =a n \\
\left(\lambda_{n}\right. & \left.=a^{n} n !\right)\end{aligned}$ \\
\hline $\begin{array}{ll}\operatorname{Gamma} \Gamma(\alpha), & (\alpha>0) \\
\frac{1}{\Gamma(\alpha)} x^{\alpha-1} e^{-x}, & x>0\end{array}$ & $\begin{aligned} \alpha_{n} & =2 n+\alpha \\
\omega_{n} & =n(n+\alpha-1) \\
\left(\lambda_{n}\right. & =n !(n+\alpha-1) \cdots \alpha)\end{aligned}$ \\
\hline Uniform on $[-1,1]$ & $\begin{aligned} \alpha_{n} & =0 \\
\omega_{n} & =\frac{n^{2}}{(2 n+1)(2 n-1)} \\
\left(\lambda_{n}\right. & \left.=\frac{(n !)^{2}}{[(2 n-1) ! !]^{2}(2 n+1)}\right)\end{aligned}$ \\
\hline $\begin{array}{l}\text { Arcsine } \\
\frac{1}{\pi \sqrt{1-x^{2}}}, \quad|x|<1\end{array}$ & $\begin{aligned} & \alpha_{n}=0 \\
& \omega_{n}= \begin{cases}\frac{1}{2}, & n=1 \\
\frac{1}{4}, & n \geq 2\end{cases} \\
&\left(\lambda_{n}=\frac{1}{2^{2 n-1}}\right)\end{aligned}$ \\
\hline $\begin{array}{l}\text { Semicircle } \\
\frac{2}{\pi} \sqrt{1-x^{2}}, \quad|x|<1\end{array}$ & $\begin{aligned} \alpha_{n} & =0 \\
\omega_{n} & =\frac{1}{4} \\
\left(\lambda_{n}\right. & \left.=\frac{1}{4^{n}}\right)\end{aligned}$ \\
\hline $\begin{array}{rr}\frac{1}{\sqrt{\pi}} \frac{\Gamma(\beta+1)}{\Gamma\left(\beta+\frac{1}{2}\right)}\left(1-x^{2}\right)^{\beta-\frac{1}{2}}, & |x|<1 \\
\beta>-\frac{1}{2}, & \beta \neq 0,1\end{array}$ & $\begin{array}{l}\alpha_{n}=0 \\
\omega_{n}=\frac{n(n+2 \beta-1)}{4(n+\beta)(n+\beta-1)}\end{array}$ \\
\hline $\begin{array}{l}\text { Negative binomial } \\
r>0,0<p<1 \\
P(X=x)=p^{r}\left(\begin{array}{c}-r \\
x\end{array}\right)(-1)^{x}(1-p)^{x}, \\
x \in \mathbb{N} \cup\{0\}\end{array}$ & $\begin{aligned} \alpha_{n} & =\frac{(2-p) n+r(1-p)}{p} \\
\omega_{n} & =\frac{n(n+r-1)(1-p)}{p^{2}} \\
\left(\lambda_{n}\right. & \left.=\frac{n !(1-p)^{n}(n+r-1) \cdots r}{p^{2 n}}\right)\end{aligned}$ \\
\hline
\end{tabular}


Table 3 .

\begin{tabular}{|c|c|}
\hline Measure & {$\left[a^{-}, a^{+}\right] P_{n}$} \\
\hline $\begin{array}{l}\text { Gaussian } \\
N\left(0, \sigma^{2}\right)\end{array}$ & $\sigma^{2} P_{n}$ \\
\hline $\begin{array}{l}\text { Poisson } \\
\text { Poi }(a)\end{array}$ & $a P_{n}$ \\
\hline $\begin{array}{ll}\operatorname{Gamma} \Gamma(\alpha), & (\alpha>0) \\
\frac{1}{\Gamma(\alpha)} x^{\alpha-1} e^{-x}, & x>0\end{array}$ & $(2 n+\alpha) P_{n}$ \\
\hline Uniform on $[-1,1]$ & $-\frac{1}{(2 n+3)(2 n+1)(2 n-1)} P_{n}$ \\
\hline $\begin{array}{l}\text { Arcsine } \\
\frac{1}{\pi \sqrt{1-x^{2}}}, \quad|x|<1\end{array}$ & 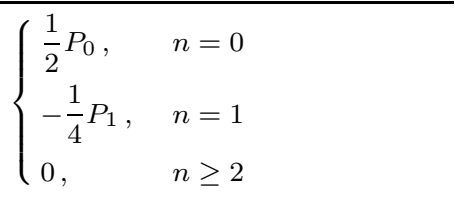 \\
\hline $\begin{array}{l}\text { Semicircle } \\
\frac{2}{\pi} \sqrt{1-x^{2}}, \quad|x|<1\end{array}$ & $\begin{cases}\frac{1}{4} P_{0}, & n=0 \\
0, & n \geq 1\end{cases}$ \\
\hline $\begin{array}{r}\frac{1}{\sqrt{\pi}} \frac{\Gamma(\beta+1)}{\Gamma\left(\beta+\frac{1}{2}\right)}\left(1-x^{2}\right)^{\beta-\frac{1}{2}}, \quad|x|<1 \\
\beta>-\frac{1}{2}, \quad \beta \neq 0,1\end{array}$ & $\frac{\beta^{2}-\beta}{2(n+1+\beta)(n+\beta)(n-1+\beta)} P_{n}$ \\
\hline $\begin{array}{l}\text { Negative binomial } \\
r>0,0<p<1 \\
P(X=x)=p^{r}\left(\begin{array}{c}-r \\
x\end{array}\right)(-1)^{x}(1-p)^{x} \\
x \in \mathbb{N} \cup\{0\}\end{array}$ & $\frac{(2 n+r)(1-p)}{p^{2}} P_{n}$ \\
\hline
\end{tabular}

From relation (4.1) we can see that the operator $a^{0}$ is determined by the parameters $\left\{\alpha_{n}\right\}_{n \geq 0}$, while the commutator operator $\left[a^{-}, a^{+}\right]$is derived from the numbers $\left\{\omega_{n}\right\}_{n \geq 1}$.

\section{Acknowledgments}

A.S. was partially supported by the NSF grant DMS 0400526.

\section{References}

1. L. Accardi and M. Bożejko, Interacting Fock space and Gaussianization of probability measures, Infin. Dimens. Anal. Quantum Probab. Relat. Topics 1 (1998) 663-670.

2. L. Accardi, H.-H. Kuo and A. Stan, Characterization of probability measures through the canonically associated interacting Fock spaces, Infin. Dimens. Anal. Quantum Probab. Relat. Topics 7 (2005) 485-505. 
3. L. Accardi, Y. G. Lu and I. Volovich, The QED Hilbert module and interacting Fock spaces, IIAS Reports No. 1997-008 (1997) International Institute for Advanced Studies, Kyoto.

4. L. Accardi and M. Nahni, Interacting Fock space and orthogonal polynomials in several variables, preprint (2002).

5. L. Accardi and M. Skeide, Interacting Fock space versus full Fock module, Volterra preprint, No. 328 (1998).

6. N. Asai, I. Kubo and H.-H. Kuo, Multiplicative renormalization and generating functions I, Taiwanese J. Math. 7 (2003) 89-101.

7. N. Asai, I. Kubo and H.-H. Kuo, Multiplicative renormalization and generating functions II, Taiwanese J. Math. 8 (2004) 593-628.

8. N. Asai, I. Kubo and H.-H. Kuo, Generating functions of orthogonal polynomials and Szegö-Jacobi parameters, Probab. Math. Stat. 23 (2003) 273-291.

9. J. A. Shohat and J. D. Tamarkin, The Problem of Moments, Mathematical Surveys, No. I (Amer. Math. Soc., 1943).

10. T. J. Stieltjes, Recherches sur les fractiones continues, Ann. Fac. Sci. Toulouse 8 (1894), T 1-122; 9 (1895) A5-47.

11. M. Szegö, Orthogonal Polynomials, Coll. Publ., Vol. 23 (Amer. Math. Soc., 1975). 